\title{
A study on evaluation strategies in dimensional $X$-ray computed tomography by estimation of measurement uncertainties
}

\author{
P. Müller ${ }^{\star}$, J. Hiller, A. Cantatore, and L. De Chiffre \\ Technical University of Denmark, Department of Mechanical Engineering, Produktionstorvet, 2800 Kgs. Lyngby, Denmark
}

Received: 29 May 2012 / Accepted: 5 July 2012

\begin{abstract}
Computed tomography has entered the industrial world in 1980's as a technique for nondestructive testing and has nowadays become a revolutionary tool for dimensional metrology, suitable for actual/nominal comparison and verification of geometrical and dimensional tolerances. This paper evaluates measurement results using different measuring strategies applied in different inspection software packages for volume and surface data analysis. The strategy influence is determined by calculating the measurement uncertainty. This investigation includes measurements of two industrial items, an aluminium pipe connector and a plastic toggle, a hearing aid component. These are measured using a commercial CT scanner. Traceability is transferred using tactile and optical coordinate measuring machines, which are used to produce reference measurements. Results show that measurements of diameter for both parts resulted in smaller systematic errors compared to distance and height measurements. It was found that uncertainties of all measurands evaluated on surface data were generally greater compared to measurements performed on volume data.
\end{abstract}

Keywords: Computed tomography; evaluation strategy; dimensional metrology; measurement uncertainty

\section{Introduction}

X-ray Computed Tomography (CT) is a non-destructive measuring technique, allowing inspection of internal and external geometries of parts. This makes nowadays CT a widely used measuring technique applied in many industrial fields, e.g. material science, electronics, medical, food, aerospace, automotive. Several industrial applications using CT have been for example reported in [1-3]. In particular, CT has become an important player in the field of coordinate metrology. This is due to the fact that by using CT, a complete three-dimensional volume model of the scanned part can be obtained in a relatively short time. Compared to other measuring techniques, e.g. tactile measuring techniques, parts scanned by CT yield high information density. However, when using CT, measurement capability is reduced due to measurement errors (e.g. image artifacts). This is one of the recent topics in CT metrology. Several studies document that artifacts in CT images have a great influence on dimensional measurements $[4,5]$. Among artifacts which mostly occur in CT images belong beam hardening, scatter radiation, ring artifacts, being the physical effects in CT. These appear in the reconstructed volume and cause, for example, problems in surface determination, and thus measurement errors. Methods dealing with corrections of these unwanted effects are being developed. An overview of image artifacts along with correction techniques is given in [1].
In general, a big number of factors influence the overall performance of CT. Studies concerning quantification of influence factors have been carried out by number of authors. Influence factors in CT can be categorized into groups, e.g. factors connected with the hardware (X-ray source, rotary table, X-ray detector), software and data processing (3D reconstruction, threshold determination and surface generation, data correction), environment (temperature, humidity), measured object (geometry, material) and operator (scanning parameters) [6-8]. Due to the big number of influence factors, an assessment of measurement uncertainty is a challenge. Traceability establishment has therefore become a key issue in CT [9]. Several studies have been done concerning uncertainty assessment. An overview of different approaches for uncertainty estimation is described in $[1,10,11]$. This includes uncertainty evaluation by expression for analytical calculated uncertainty budget (standard GUM method [12]), experimental methods (e.g. use of calibrated workpieces according to 15530-3 [13]), theoretical methods (e.g. MonteCarlo simulation [14]) and combination of these methods. In [6], the authors focused on the influence of the operator on dimensional measurements. They developed an equation characterizing the measurement CT process and calculated the measurement uncertainty according to the GUM. In [15], the authors calculated the measurement uncertainty using experimental methods. In particular, they followed ISO 15530-3 describing the use of calibrated

^ Correspondence: pavm@mek.dtu.dk 
Table 1. An overview of software packages for measurements on volume/surface data.

\begin{tabular}{cc}
\hline Software tool & Data set \\
\hline SW1 & Voxel model/volume data \\
SW2 & Voxel model/volume data \\
SW3 & STL model/surface data \\
\hline
\end{tabular}

workpieces. The Monte Carlo method for uncertainty estimations in CT was used in $[4,16]$.

By CT, a complete volumetric 3D model of a scanned part is obtained, both as a volume model or a surface model, defined from the volume model by creating a polygonal mesh. Software tools, devoted to manage either of the models, are used for dimensional measurements and other metrological applications. However, each software tool provides different algorithms for application of measuring strategies to define selected measurands. In the present investigation, three software tools were used. The objective of this study is to perform geometrical measurements on industrial parts using CT and to quantify the influence of application of different measuring strategies on selected geometrical features by the assessment of the measurement uncertainty.

\section{Case description}

Two industrial parts were selected, each made of different material and of different size. The reason for choosing two such different objects is to point out some of the specific characteristics of $\mathrm{CT}$ in terms of material.

The first object is an aluminium alloyed pipe connector, manufactured by cold forging and subsequently machined to desired dimensions. This part is used in automotive industry. Four measurands shown in (Fig. 1 left and middle) (two dimensional and two geometrical) were defined: inner diameter of the hole $\left(d_{P}\right)$, distance between two parallel surfaces of the inner flange $\left(L_{P}\right)$, parallelism between the two surfaces $\left(P_{P}\right)$ and cylindricity $\left(C_{P}\right)$ of the inner hole. The second part is a polymeric micro component used for a hearing aid applications, a toggle, produced by polymer injection moulding and is made of liquid crystal polymer (LPC) with a part weight of $35 \mathrm{~g}$. Four measurands shown in (Fig. 1 right) (three dimensional and one geometrical) were defined according to $[17,18]$. These are: outer diameter of the toggle $\left(D_{T}\right)$, inner diameter of the hole in the middle of the part $\left(d_{T}\right)$, concentricity defined between the hole in the middle of the part and the outer cylindrical feature $\left(C_{T}\right)$, and height $\left(H_{T}\right)$ of the pillar.

Dimensional and geometrical measurements were performed both on volume and surface data (polygonal mesh). Three commercial software packages for CT analysis were used and are summarized in Table 1. Each of the software offers different algorithms and measuring strategies for fitting geometrical primitives on the $3 \mathrm{D}$ models.

\section{Measuring setup for tactile, optical and $C T$ measurements}

Before both parts were measured using CT, they were first calibrated using tactile and optical coordinate measuring machines (CMM). These measurements were considered as reference, ensuring measurement traceability. This is due to the fact that measurements performed using these technologies are well accepted. CT measurements are considered as actual measurements. In this study, the reference and actual measurements are not compared, however, a difference between the two is taken into account for uncertainty estimation.

\subsection{Tactile reference measurements}

The pipe connector was measured using a Zeiss OMC 850 tactile CMM with stated maximum permissible error $\mathrm{MPE}=(3+L / 250) \mu \mathrm{m}(L$ in $\mathrm{mm})$. Measurements were performed in a temperature controlled laboratory with temperature of $20 \pm 0.5{ }^{\circ} \mathrm{C}$. Measurements carried out using the tactile CMM were realized using three styli with corresponding number of probes. The nominal dimensions (diameter, $\varnothing$ and length, $l$, of styli) are: 1) $\varnothing 3.0 \mathrm{~mm}$, $l=58 \mathrm{~mm}$ (axial), 2) Ø1.5 mm, $l=56 \mathrm{~mm}$ (horizontal) and 3) $\varnothing 5.0 \mathrm{~mm}, l=53 \mathrm{~mm}$ (horizontal), configured so that measurement in all directions was possible without repositioning of the workpiece. All the measurements were repeated three times.

\subsection{Optical reference measurements}

The toggle was calibrated according to procedures described in $[17,18]$, first using a high accuracy Zeiss tactile $\mathrm{CMM}$ with $\mathrm{MPE}=(0.4+L / 900) \mu \mathrm{m}(L$ in $\mathrm{mm})$ and secondly by Schut DeMeet 220 optical CMM, yielding accuracy of $\mathrm{MPE}=(4+L / 150) \mu \mathrm{m}(L$ in $\mathrm{mm})$ in $X$ and $Y$ direction and $3.5 \mu \mathrm{m}$ in $Z$ direction Calibration values from the optical CMM were considered in this study.

\subsection{CT measurements}

Both parts were then scanned using a Zeiss Metrotom 1500 cone beam CT scanner. Measurements performed using the CT scanner were reproduced three times. The reproducibility was assessed by scanning the parts in different days and repositioning of the parts from the fixture. For reliable statistics the number of CT measurements is not appropriate, however, the approach presented in this work is more industrial-like, thus the authors found the number of measurements of each part adequate. Both parts were freely placed in a fixture made of polystyrene (PS), however prevented from any movement during the rotation.

Scanning parameters chosen by the operator are shown in Table 2. The choice of different scanning parameters for both parts is due to their different size, material, shape, 

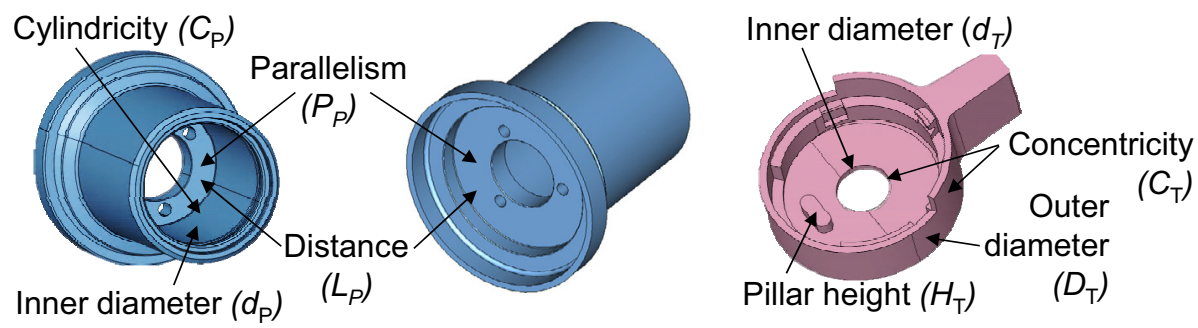

Fig. 1. Pipe connector (left and middle) and Toggle (right). The selected measurands are indicated.

Table 2. An overview of the scanning parameters.

\begin{tabular}{lccc}
\hline Parameter & Unit & Pipe connector & Toggle \\
\hline Voltage & $\mathrm{kV}$ & 210 & 130 \\
Current & $\mu \mathrm{A}$ & 500 & 150 \\
Focal spot size & $\mu \mathrm{m}$ & 105 & 19 \\
X-ray filter & - & $\mathrm{Cu} 0.25 \mathrm{~mm}$ & - \\
Detector matrix & pixel & $1024 \times 1024$ & $1024 \times 1024$ \\
Pixel size & $\mu \mathrm{m}$ & 400 & 400 \\
Integration time & $\mathrm{ms}$ & 1000 & 1000 \\
Magnification & - & 3.7 & 20.8 \\
Voxel size & $\mu \mathrm{m}$ & 108 & 19 \\
No. of projections & - & 720 & 720 \\
\hline
\end{tabular}

etc. It can be for example noticed that the toggle allows much higher magnification for its smaller size and therefore yields higher resolution in terms of smaller voxel size compared to the pipe connector. In case of the pipe connector, a copper filter $0.25 \mathrm{~mm}$ thick was applied to minimize beam hardening effect [5]. Filters are not necessary for scanning of plastic parts. The focus spot size is a result of selected X-ray tube power. It can be noticed that employing higher power (voltage and current) leads to increase of the focus spot size, and thus to increased image blurring. Higher power applied when scanning the aluminium pipe connector is due to higher material density and greater wall thickness. Higher power increases the radiation intensity and the penetration of the photons through the matter.

CT measurements were performed in a temperature controlled laboratory with temperature of $21 \pm 0.5{ }^{\circ} \mathrm{C}$. The temperature in the CT scanner was observed to be $22 \pm 0.5^{\circ} \mathrm{C}$ in different days when the parts were scanned.

\section{Uncertainty assessment}

According to GUM, the uncertainty is a parameter associated with the result of a measurement that characterizes the dispersion of the values that could reasonably be attributed to the measurand. Statement about measurement uncertainty enables users to make comparisons, quantify quality of measurement and is important for decision making in terms of tolerance verification.

\subsection{Uncertainty estimation for tactile measurements}

The measurement uncertainties for tactile measurements $U_{\text {ref }}$ of the pipe connector were calculated according to a simplified uncertainty budget - PUMA method (ISO 14253-2) [19], as described in equation (1).

$$
U_{\text {ref }}=k \sqrt{u_{i}^{2}+u_{p}^{2}+u_{e}^{2}}
$$

where $k$ is coverage factor $(k=2$ for a confidence level of $95 \%), u_{\mathrm{i}}$ is standard calibration uncertainty of the measuring instrument, taking into account the MPE of the machine, calculated as $u_{i}=\mathrm{MPE} / 2, u_{\mathrm{p}}$ is standard uncertainty of the measuring procedure, calculated as $u_{\mathrm{p}}=h(s / \sqrt{ } n)$, where $h$ is safety factor $(h=2.3$ for three measurements), $s$ is standard deviation of three repeated measurements and $n$ is number of measurements $(n=3)$, $u_{\mathrm{e}}$ is temperature-related standard uncertainty calculated for a deviation of $\pm 0.5^{\circ} \mathrm{C}$ and using a coefficient of linear expansion for aluminum of $23 \times 10^{-6}{ }^{\circ} \mathrm{C}^{-1}$.

\subsection{Uncertainty estimation for optical measurements}

Uncertainty assessment of measurements of polymer parts with a high accuracy tactile CMM was based on ISO 15530-3 [20]. Subsequently, the calibration data obtained from tactile measurements is employed to calculate the uncertainty of optical CMM measurements, which are used in this work. A detailed uncertainty budget is discussed in [18].

\subsection{Uncertainty estimation for CT measurements}

The measurement uncertainty $U_{C T}$ of both parts measured using the CT scanner was calculated according to ISO $15530-3$ [13] as described in equation (2).

$$
U_{C T}=k \sqrt{u_{\mathrm{ref}}^{2}+u_{p}^{2}+u_{e}^{2}+b^{2}}
$$

where $k$ is coverage factor ( $k=2$ for a confidence interval of $95 \%), u_{\text {ref }}$ is standard uncertainty as previously calculated for tactile and optical measurements $\left(u_{\text {ref }}=U_{\text {ref }} / 2\right)$, $u_{p}$ is standard uncertainty of the measuring procedure for each measurand, calculated as $u_{p}=h(s / \sqrt{ } n)$, where $h$ is safety factor $(h=2.3), s$ is standard deviation of three reproduced measurements and $n$ is number of measurements 


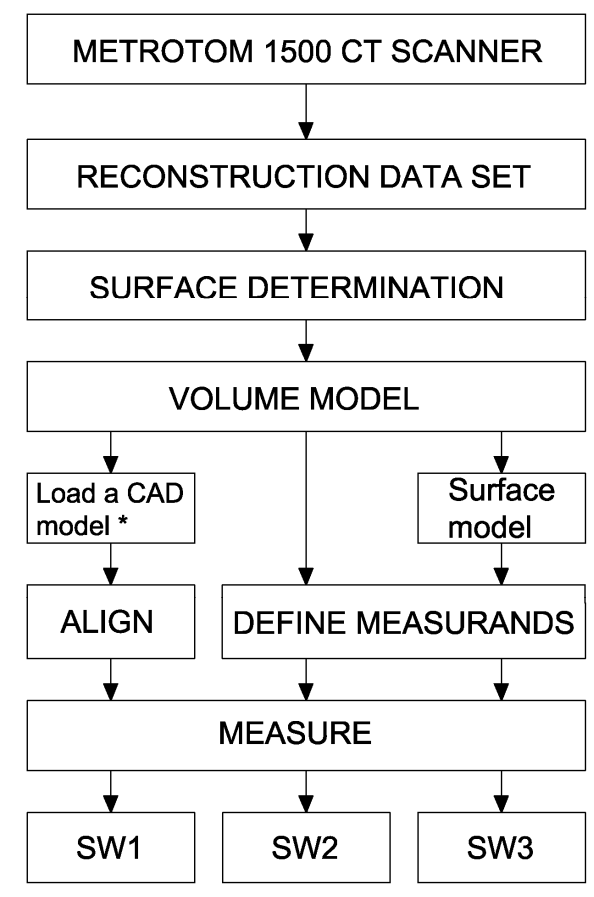

Fig. 2. Measuring procedure for selected software. ${ }^{*} \mathrm{CAD}$ model with already programmed measurement plan.

$(n=3), u_{e}$ is temperature-related standard uncertainty calculated for a deviation of $\pm 0.5^{\circ} \mathrm{C}$ and using a coefficient of linear expansion for aluminum of $23 \times 10^{-6}{ }^{\circ} \mathrm{C}^{-1}$ and $49 \times 10^{-6}{ }^{\circ} \mathrm{C}^{-1}$ for LPC, $b$ is systematic error calculated as a difference between measurements performed using CT scanner and tactile CMM and optical CMM respectively.

Standards (e.g. GUM) suggest to correct first for systematic errors (bias) and then to calculate the measurement uncertainty. Due to the fact that in CT many influence factors like scale errors, errors due to the focus spot drift and other unknown factors have an effect on geometrical measurements, and their quantification is rather a difficult task, we do include these systematic errors for estimation of measurement uncertainty in this work. Bias $b$ is determined empirically and as such is treated as random errors and therefore has to be added squared under the radical, just like other uncertainty contributors [21]. Assessment of systematic errors is important, showing the actual difference between the two measuring machines.

\section{Process chain for data evaluation and definition of measuring strategies}

The focus of this investigation is to perform measurements on simple geometrical features, i.e. cylinders, circles, planes. These are features where a single outlier, measured point outside the specified range, will not influence the overall measurement result.

A process chain for measurements of both parts using three software packages is schematically shown in (Fig. 2).
Table 3. An overview of evaluation strategies for selected measurands applied in the software packages. Approximately 1000 measured points were used to define a respective geometrical feature.

\begin{tabular}{llll}
\hline Measurand & SW1 & SW2 & SW3 \\
\hline \multirow{2}{*}{ Diameter $\left(d_{P}, D_{T}\right)$} & Circle & Circle & Circle \\
& Recall & Feature fit & Feature fit \\
\hline Diameter $\left(d_{T}\right)$ & Circle & Circle & Circle \\
\hline Distance $\left(L_{P}\right)$ & Plane-Plane & Plane-Plane & Plane-Plane \\
and Height $\left(H_{T}\right)$ & Point-Plane & Point-Plane & Point-Plane \\
\hline \multirow{2}{*}{ Cylindricity $\left(C_{P}\right)$} & Spiral & Feature fit & Feature fit \\
& Recall & Cylinder circle & Cylinder circle \\
\hline \multirow{2}{*}{ Concentricity $\left(C_{T}\right)$} & Circle & Circle & Circle \\
& Recall & Feature fit & Feature fit \\
\end{tabular}

The evaluation method for fitting geometrical primitives is least square method (also called Gaussian best fit).

After scanning of the parts, 3D reconstruction and surface determination, a 3D volume model is visualized in SW1 and SW2. In SW1, a surface is determined on the reconstructed part using automatically generated "optimal" threshold. Then, a CAD model with already defined measuring strategies on selected measurands is imported and aligned with the volume model using a best fit method. In SW2, the reconstructed part is visualized, the surface is determined on the part using a local adaptive threshold method and measurements are performed by defining measurands directly on the volume model. A surface model in the form of triangulated mesh (STL) is generated in SW1 and imported in SW3. Here, measurements are performed by defining measurands on the STL model.

Different measuring strategies for diameter, height, distance, cylindricity and concentricity measurements for both parts under study were applied in each of the software. Table 3 presents an overview of measuring strategies used to determine above mentioned measurands. It can be noticed that some measuring strategies are common to all software packages and some are different. This is due to various fitting algorithms which individual software packages are equipped with. The definition of the measurands is specified as follows:

\section{Diameter (Pipe connector and Toggle)}

- Circle: measurement is performed at different levels with respect to the position of a reference plane by fitting a respective number of circles. Diameter based on a least-square method is then calculated as an average of the respective number of circles.

- Feature fit: by selecting a feature (in our case a cylindrical surface), a least square cylinder is created on the surface of the respective feature. The diameter is the one of the least square cylinder. 
a) Diameter, $d_{p}$

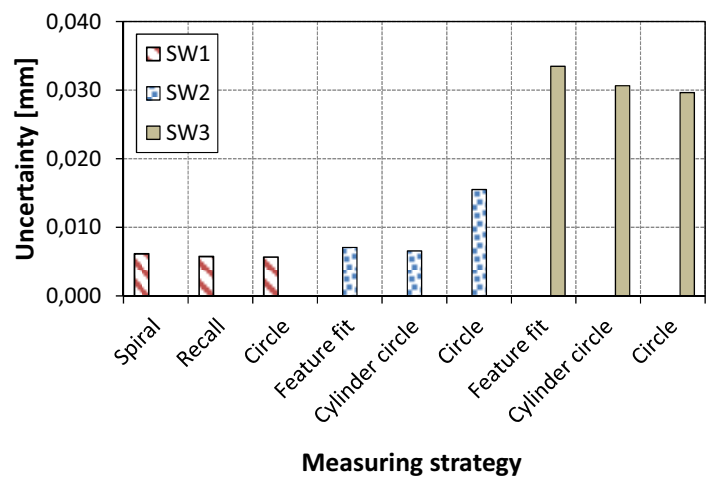

c) Cylindricity, $C_{\mathrm{p}}$

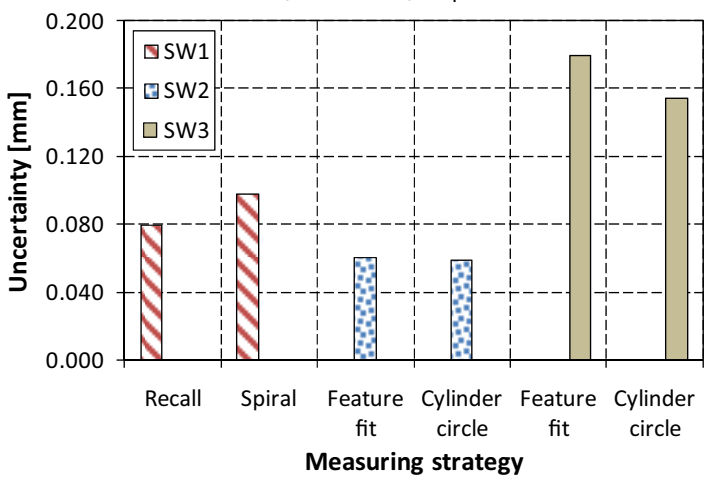

b) Distance, $L_{p}$

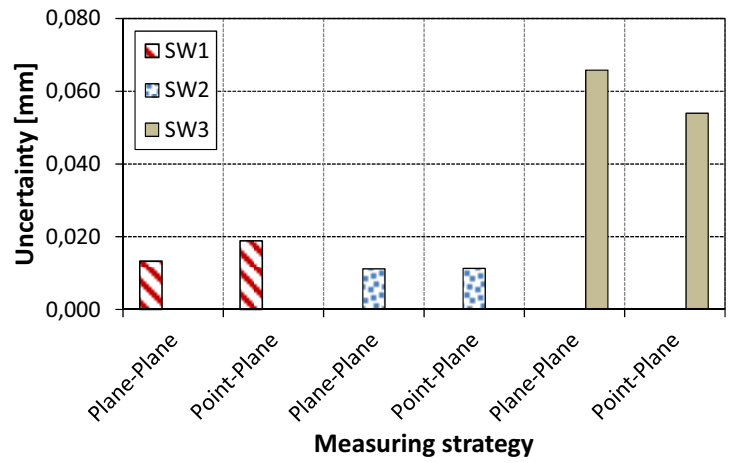

d) Parallelism, $P_{\mathrm{p}}$

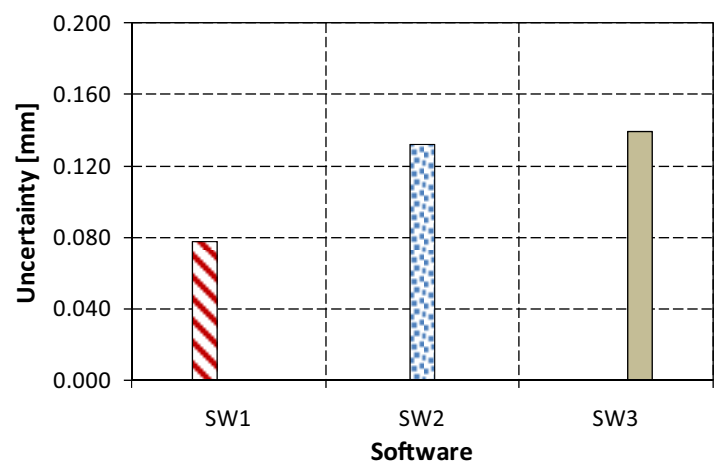

Fig. 3. Expanded uncertainties at $95 \%$ confidence level for measurements performed on the pipe connector. Measurement uncertainties are calculated according to equation (2).

- Cylinder circle: by selecting points in circular crosssections (in planes perpendicular to the axis of a cylinder) at two levels with respect to the position of a reference plane, the cylinder is fit in between these levels. Diameter is then given by the least square fit in the specified range.

- Spiral: a spiral is fit on the cylinder with defined number of revolutions and number of points. Diameter based on a least-square method is then calculated.

- Recall: recalls previously created features (in our case - circles). Diameter is then calculated as an average value of both circles.

\section{Distance (Pipe connector) and height (Toggle)}

- Plane-Plane: by selecting surfaces, best fit planes are fit. The distance is then calculated by projecting the center point of the fit plane onto the other plane in normal direction.

- Point-Plane: by selecting single points on one surface and fitting a plane on the other surface with respect to which the distance/height is to be calculated, the distance is calculated by projecting the fit points onto the plane in normal direction.

Further description of measurands assessment is given in $[22]$.

\section{Results and discussion}

\subsection{Pipe connector}

Results of the uncertainty calculation are presented in (Fig. 3). Measurement uncertainties in the figure are calculated according to equation (2). Generally, one can observe that bigger uncertainties for selected measurands are associated with measurements carried out using SW3. One of the reasons why this happens may be the fact that measurements in this software were done on a polygonal mesh. It is mentioned in [23] that measurements performed on the polygonal mesh result in worsened quality and measurement inaccuracy, because the number of triangles on the polygonal mesh has to be optimized so that a software tool is able to handle the mesh. By decimating the number of triangles in the extracted polygonal mesh the number of measured points is reduced. This is further connected with the existence of noise which is present at some parts of the volume model. (Fig. 4) shows, for example, a reconstructed slice, further modified in Fiji software (a free software for image analysis) to enhance the appearance of noise, occurring at the top where this part of the object was at the borders of the detector, and at the bottom where the length of the X-rays traveling through the aluminum matter of the pipe connector was big. These are common problems when using $\mathrm{CT}$. In order to reduce the noise, it is advisable to position the workpiece on the rotary table so that the length the X-rays travel through the 


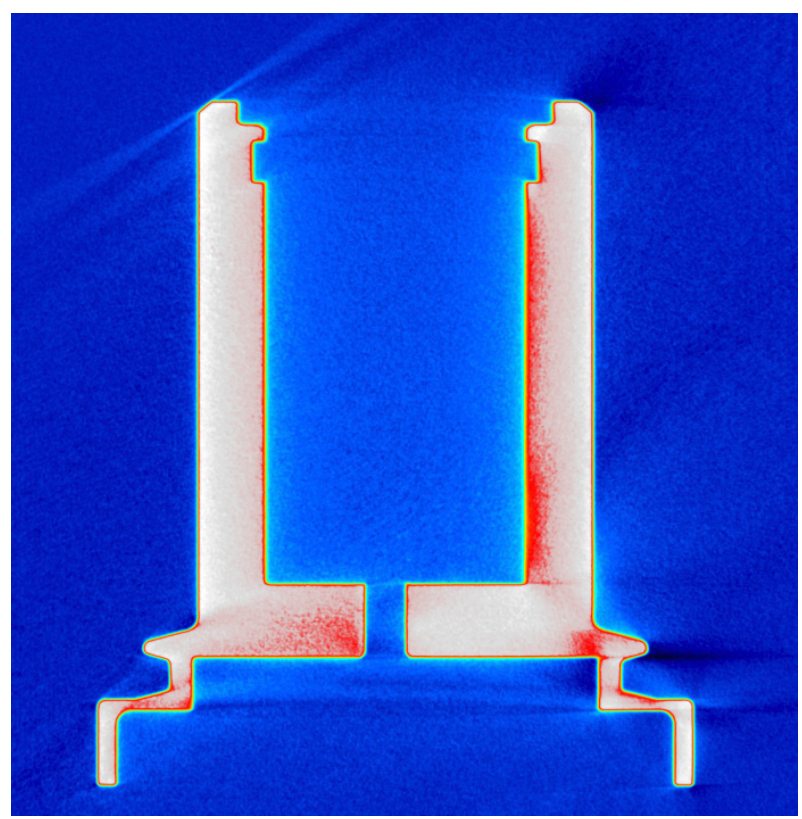

Fig. 4. Reconstruction slice of the pipe connector showing image artifacts at the top (object at the borders of the detector), and at the bottom (big length of the X-rays traveling through the aluminum matter).

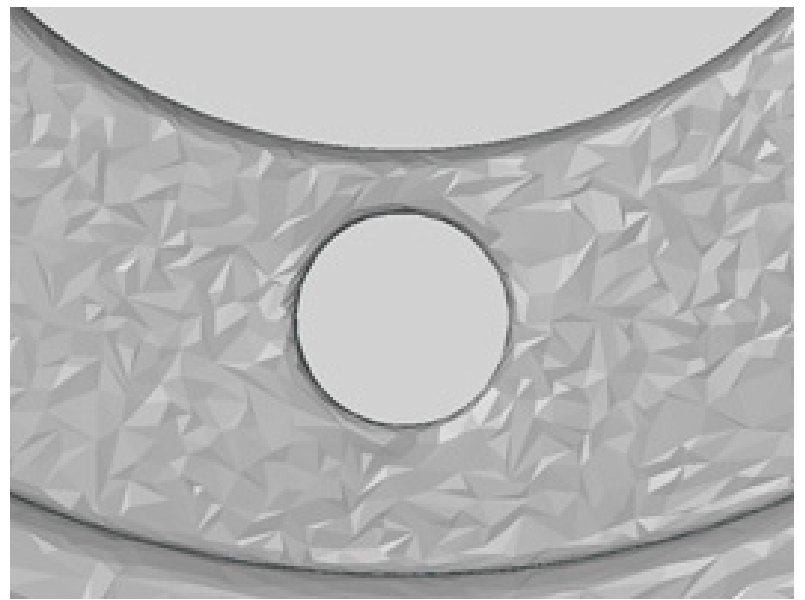

Fig. 5. A polygonal mesh (STL) created on the pipe connector.

matter is minimized. The pipe connector was positioned at approximately $45^{\circ}$ in our case; however the length of the X-rays which travelled across the part was big enough to cause noise (image artifacts). Generally, STL data is very sensitive regarding image noise. So, when a polygonal mesh is created on the volume model with noise, this noise becomes a part of the mesh (Fig. 5). A colour map of deviations between volume and surface models is shown in (Fig. 6). Here, the maximum deviations between the two models occur where the radiographic lengths are big.

For diameter measurements the uncertainties calculated using SW1 and SW2 are in the same range as uncertainties obtained with reference measurements. This is due to more robust fitting algorithms applied for diameter evaluation (Fig. 3a) rather than for measurements of

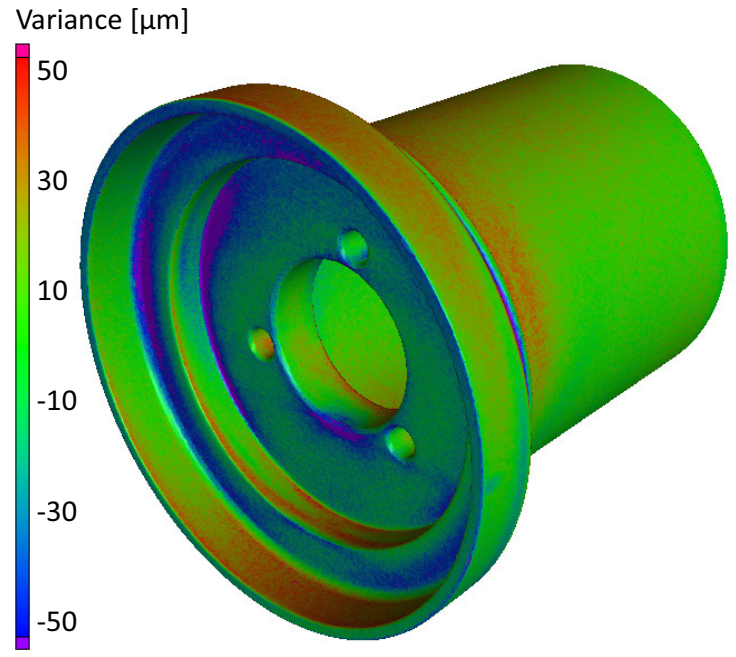

Fig. 6. Colour map of the pipe connector showing deviations between a volume model and a surface model (STL).

distance between planes (Fig. 3b). That is because bidirectional measurements (in our case distance between two parallel surfaces of the inner flange) in CT are greatly influenced by the noise which is threshold sensitive. Uncertainties calculated for measurements of diameter and distance in SW3 are greater than 100\% compared to uncertainties calculated for measurements in SW1 and SW2, which confirms the problematic concerning measurements on the polygonal mesh. Considering individual software tools, the selection of measuring strategies for diameter and height measurements seems not to be significant. Small variations among measurement uncertainties calculated for different measuring strategies are naturally due to different fitting algorithms, as explained in Section 5, however, the difference is negligible. For example, for diameter measurements $d_{P}$ in SW2, values obtained using measuring strategy "Circle" are double compared to other strategies. As mentioned in Section 5, this measuring strategy is based on measurements of circles at different levels, and so this strategy is more sensitive to deviations with respect to the reference measurements. Moreover, alignment could be a critical point here, since the alignment was defined on a part of the object where noise occurred (i.e. a plane defined on the inner flange). Comparing the two software tools for analysis of volume data sets, SW1 and SW2, difference between measurement uncertainties is smaller than $8 \mu \mathrm{m}$. Moreover, uncertainties related to diameter and distance measurements are calculated in a reasonable range for CT measurements. Uncertainties related to geometrical tolerances, cylindricity and parallelism, (Figs. 3c and 3d), respectively, are bigger compared to measurements of diameter and distance. This is due to the fact that measurements of geometrical features are more problematic and lead to bigger errors in CT [24]. Uncertainties related to measurements of cylindricity and parallelism are, again, bigger for measurements on surface data. Uncertainties calculated for parallelism tolerance are 
Table 4. Bias contribution to the measurement uncertainty $\left(|b| / U_{\mathrm{CT}}\right.$ in \%) calculated for the pipe connector.

\begin{tabular}{ccccc}
\hline \multirow{2}{*}{ Measurand } & \multirow{2}{*}{ Measuring strategy } & \multicolumn{3}{c}{ Software tool } \\
& SW1 & SW2 & SW3 \\
\hline \multirow{4}{*}{$d_{P}$} & Circle & 3 & 47 & 44 \\
& Feature fit & & 29 & 46 \\
& Cylinder circle & & 31 & \\
& Spiral & 17 & & \\
& Recall & 1 & & \\
\hline \multirow{2}{*}{$L_{P}$} & Plane-Plane & 29 & 32 & 41 \\
& Point-Plane & 23 & 25 & 46 \\
\hline$P_{P}$ & & 47 & 48 & 50 \\
\hline \multirow{2}{*}{$C_{P}$} & Feature fit & & 50 & 49 \\
& Spiral & 49 & & \\
\hline
\end{tabular}

approx. $80 \mu \mathrm{m}$ and greater, possibly showing that noise in the region, where this feature was measured, was critical.

It was investigated that the bias was dominant uncertainty contributor for measurements on the surface data and for measurements of the geometrical tolerances. Table 4 summarizes results of bias contribution to the measurement uncertainty, expressed by a ratio $|b| / U_{\mathrm{CT}}(b$ is bias and $U_{\mathrm{CT}}$ is expanded measurement uncertainty). The bias is a critical factor and for this reason, it is therefore recommended to carry out reference measurements on the features which can be measured (for example tactilely) and to take this factor into account for calculation of the measurement uncertainty in $\mathrm{CT}$.

\subsection{Toggle}

Results of the uncertainty calculation are presented in (Fig. 7). Measurement uncertainties for CT measurements are assessed according to equation (2). Generally, bias (its contribution to the expanded uncertainty is summarized in Tab. 5) was found dominating uncertainty contributor for measurements of inner diameter $d_{T}$, height of the pillar $H_{T}$ and concentricity $C_{T}$, being in the range from 13 to $32 \mu \mathrm{m}$. This was the same for all three software tools. For measurements of the outer diameter $D_{T}$, the maximum bias value of $4 \mu \mathrm{m}$ was obtained for measurements in SW3, in SW1 and SW2 the bias was $1 \mu \mathrm{m}$. Low bias values for outer diameter measurements of the toggle are in good agreement with measurements of the pipe connector as discussed in Section 6.1, and confirms that measurements of diameters, where selection of measured points is well defined, is robust. Uncertainties calculated for measurements of outer diameter using SW1 and SW2 are in agreement with reference measurements. Uncertainties calculated for measurements in SW3 are approximately double.

As bigger measurement uncertainties for most of the measurands of the pipe connector were connected with measurements in SW3, it was investigated that this was not the case for measurements of the toggle (except for outer diameter measurements). Measurement uncertain-
Table 5. Bias contribution to the measurement uncertainty $\left(|b| / U_{\mathrm{CT}}\right.$ in \%) calculated for the toggle.

\begin{tabular}{ccccc}
\hline \multirow{2}{*}{ Measurand } & Measuring strategy & \multicolumn{3}{c}{ Software tool } \\
& & SW1 & SW2 & SW3 \\
\hline \multirow{2}{*}{$D_{T}$} & Circle & 5 & 10 & 32 \\
& Feature fit & & 10 & 30 \\
& Cylinder circle & & 10 & \\
& Spiral & 18 & & \\
& Recall & 0 & & \\
\hline$d_{T}$ & Circle & 50 & 39 & 50 \\
\hline \multirow{2}{*}{$H_{T}$} & Plane-Plane & 47 & 47 & 47 \\
& Point-Plane & 47 & 47 & 47 \\
\hline \multirow{2}{*}{$C_{T}$} & Circle & 50 & 47 & 50 \\
& Feature fit & & 47 & 47 \\
& Cylinder circle & & 48 & \\
& Spiral & 50 & & \\
\hline & Recall & 50 & & \\
\hline
\end{tabular}

ties related to height and concentricity were calculated within $11 \mu \mathrm{m}$ and $6 \mu \mathrm{m}$, respectively, considering all three software tools. This can be explained by the material of the part itself. Low density materials yield high penetration rates (low attenuation of X-rays) and therefore allow more photons to be detected with the X-ray detector. Small parts are also preferable, high resolution CT scans due to their small voxel sizes are obtained, and occurrence of image artifacts is minimized.

Uncertainties calculated for measurements of the inner diameter $d_{T}$ in SW2 are smaller $(33 \mu \mathrm{m})$ compared to other two software tools (approx. $60 \mu \mathrm{m}$ ). The reason for this is, again, big contribution due to the bias. Measurements of $d_{T}$ were complicated due to its rather poor quality manufactured edge, and thus difficulties when measured. Therefore, measuring strategy had to be slightly adapted to measure this feature.

The selection of measuring strategies for all selected measurands in different software packages seems not to be significant, only small variations can be observed. These are again due to different fitting algorithms.

It was investigated that bias was dominating uncertainty contributor for measurements on both volume and surface data.

\section{Conclusions}

This paper evaluates results obtained by CT measurements of two industrial parts using different measuring strategies applied in different inspection software. The strategy influence is determined by calculating the measurement uncertainty. The items were measured using a commercial CT scanner. Reference measurements were performed on tactile and optical CMMs. Some conclusions from this investigation can be drawn and are summarized in the following:

- Diameter measurements of cylindrical features for both aluminum and plastic parts resulted in small bias and 
a) Diameter, $D_{\mathrm{T}}$

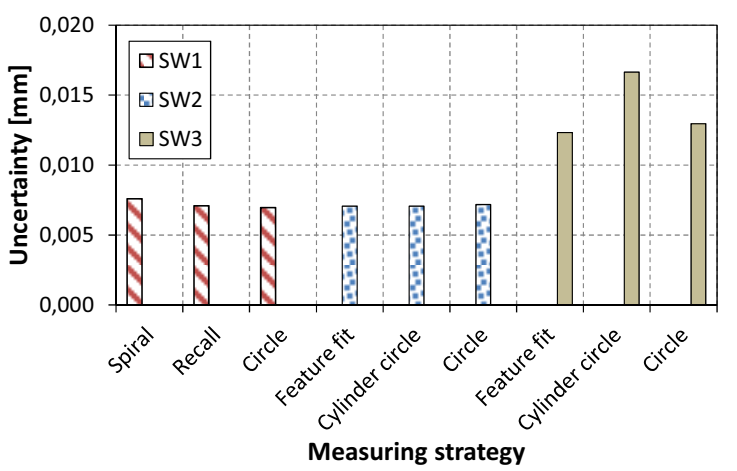

c) Height, $H_{\mathrm{T}}$

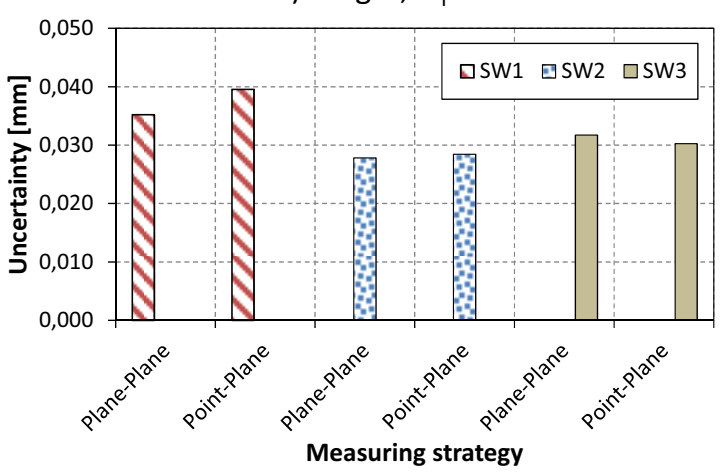

b) Diameter, $d_{\mathrm{T}}$

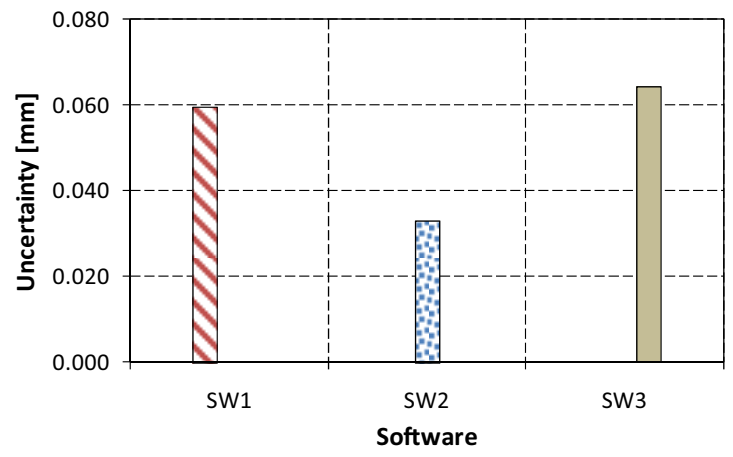

d) Concentricity, $C_{\mathrm{T}}$

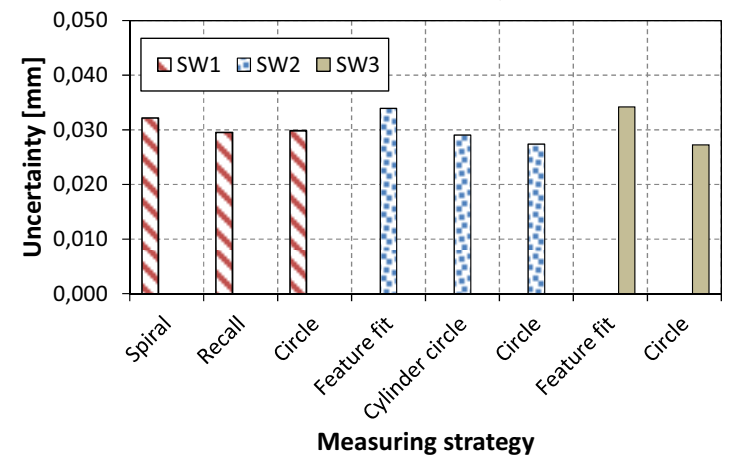

Fig. 7. Expanded uncertainties at $95 \%$ confidence level for measurements performed on the toggle. Measurement uncertainties are calculated according to equation (2).

low measurement uncertainties compared to distance and height measurements. This was due to a robust fitting of well-defined geometrical features.

- Bias as well as measurement uncertainties calculated for measurements using SW3 for the pipe connector were generally bigger compared to measurements using SW1 and SW2. This was due to the fact that measurements carried out in SW3 were done on the surface data (STL), generally resulting in worsened quality and impression in measurement due to the polygonal mesh created on the surface. In contrast, for the toggle, bias as well as measurement uncertainties were calculated in the same range for all the three software packages, except for outer diameter measurements (these were smaller).

- It was investigated that the choice of a specific measuring strategy applied to measure different features does not play a major role in our case and it is therefore a free choice for the operator who will not significantly influence the final result from CT measurements. Small variations in the choice of measuring strategy were however observed.

- This paper presents a case study only. Greater variability of parts, i.e. parts of different materials, different geometries and sizes should be considered to generalize the influence of measuring strategies on measurement uncertainty. The same concerns dimensional and geometrical tolerances, i.e. not only those tolerances used in our case should be considered to quantify effects connected with CT measurements, but other should be taken into account.

- In the case of a presence of image noise on the CT data set, one can filter these data before applying the surface (STL). One should however be careful since this may lead to degradation of the original data set and therefore significantly change shape of a part and therefore obtain different measurement result. Another possibility how to avoid noise is to change the scanning parameters (e.g. integration time, current), which is in many cases rather difficult task.

Acknowledgements. This paper discusses an investigation which was undertaken in the context of the Danish project "CIA-CT - Center for Industrial Application of CT scanning". The authors would like to thank Danish Technological Institut for kindly providing access to the CT scanner, namely to Mr. Peder Pedersen for making the CT scans. Novo Nordisk $\mathrm{A} / \mathrm{S}$ is acknowledged for kindly providing use of one of the software. Thanks to Mr. Jakob Rasmussen (Technical University of Denmark) in connection with CMM measurements.

\section{References}

1. J.-P. Kruth, M. Bartscher, S. Carmignato, R. Schmitt, L. De Chiffre, A. Weckenmann, Computed tomography for dimensional metrology, CIRP Ann. Manuf. Technol. 60, 821-842 (2011) 
2. W. Bauer, F.T. Bessler, E. Zabler, R.B. Bergmann, Computer tomography for nondestructive testing in the automotive industry, Developments in X-ray Tomography IV, edited by U. Bonse, Proc. SPIE 5535, 464-472 (2004)

3. P. Müller, R.A. Pacurar, L. De Chiffre, A. Cantatore, P. Berce, Geometrical metrology on silicone rubber by computed tomography, Proceedings of 11th Euspen International Conference, Como, Italy, 2011, pp. 243-246

4. S. Kasperl, J. Hiller, M. Krumm, Computed tomography metrology in industrial research and development, MP Mater. Test. 51, 405-411 (2009)

5. M. Krumm, S. Kasperl, M. Franz, Reducing non-linear artifacts of multi-material objects in industrial 3D computed tomography, NDT \& E Int. 41, 242-251 (2008)

6. A. Weckenmann, P. Krämer, Predetermination of measurement uncertainty in the application of computed tomography, 11th CIRP International Conference on Computer Aided Tolerancing (CAT), Annecy, France, 2009, pp. 317-330

7. F. Welkenhuyzen, K. Kiekens, M. Pierlet, W. Dewulf, P. Bleys, J.-P. Kruth, A. Voet, Industrial Computer Tomography for Dimensional Metrology: Overview of Influence Factors and Improvement Strategies, 4th International Conference on Optical Measurement Techniques for Structures and Systems (OPTIMESS), Antwerp, Belgium, 2009, pp. 401-410

8. M. Bartscher, U. Hilpert, J. Goebbels, G. Weidemann, Enhancement and proof of accuracy of industrial computed tomography (CT) measurements, CIRP Ann. Manuf. Technol. 56, 495-498 (2007)

9. S. Carmignato, D. Dreossi, L. Mancini, F. Marinello, G. Tromba, E. Savio, Testing of X-ray microtomography systems using a traceable geometrical standard, Meas. Sci. Technol. 20, 084021 (2009)

10. P. Wenig, S. Kasperl, Examination of the Measurement Uncertainty on Dimensional Measurements by X-ray Computed Tomography, Proceedings of 9th European Conference on Non-Destructive Testing (ECNDT), Berlin, Germany, 2006

11. M. Bartscher, M. Neukamm, U. Hilpert, U. NeuschaeferRube, F. Härtig, K. Kniel, K. Ehrig, A. Staude, J. Goebbels, Achieving traceability of industrial computed tomography, Key Eng. Mater. 437, 79-83 (2010)

12. ISO/IEC Guide 98-3: 2008 - Uncertainty of measurement - Part 3: Guide to the expression of uncertainty in measurement (GUM, 1995)

13. ISO 15530-3: 2009 - Geometrical product specifications (GPS) - Coordinate measuring machines (CMM): Technique for determining the uncertainty of measurement - Part 3: Use of calibrated workpieces or measurement standards
14. ISO, GUM, Suppl. 1, JCGM 101, Evaluation of Measurement Data - Supplement 1 to the "Guide to the Expression of Uncertainty in Measurement" - Propagation of Distributions Using a Monte Carlo Method (2008)

15. R. Schmitt, Ch. Niggemann, Uncertainty in measurement for X-ray-computed tomography using calibrated workpieces, Meas. Sci. Technol. 21, 1-9 (2010)

16. J. Hiller, Abschätzung von Unsicherheiten beim dimensionellen Messen mit industrieller RöntgenComputertomographie durch Simulation, Dissertation, University of Freiburg i. Br., 2011

17. S. Gasparin, G. Tosello, H.N. Hansen, M.B. Jørgensen, Quality control of injection moulded micro mechanical parts, Proceedings of 5th International Conference on Multi-Material Micro Manufacture (4M/ICOMM), Karlsruhe, Germany, 2009, pp. 175-178

18. G. Tosello, H.N. Hansen, S. Gasparin, Applications of dimensional micro metrology to the product and process quality control in manufacturing of precision polymer micro components, CIRP Ann. Manuf. Technol. 58, 467-472 (2009)

19. ISO 14253-2: 2011 - Geometrical product specifications (GPS) - Inspection by measurement of workpieces and measuring equipment - Part 2: Guidance for the estimation of uncertainty in GPS measurement, in calibration of measuring equipment and in product verification

20. ISO/TS 15530-3: 2004 Geometrical Product Specifications (GPS) - Coordinate Measuring Machines (CMM): Technique for Determining the Uncertainty of Measurement - Part 3: Use of Calibrated Workpieces or Standards, i-v, pp. 1-16

21. F. Härtig, M. Krystek, Correct treatment of systematic errors for the evaluation of measurement uncertainty, Proceedings of 9th International Symposium on Measurement Technology and Intelligent Instruments (ISMTII), Saint Petersburg, 2009, Vol. 1, pp. 1-016-1-019

22. P. Müller, J. Hiller, A. Cantatore, L. De Chiffre, Investigation of measuring strategies in computed tomography, International Conference on Advanced Manufacturing Engineering (NEWTECH), Brno, Czech Republic, 2011, pp. $31-42$

23. C. Reinhart, C. Poliwoda, T. Guenther, W. Roemer, S. Maass, C. Gosch, Modern voxel based data and geometry analysis software tools for industrial CT, $C D-R O M$ Proceedings of 16th WCNDT - World Conference on NDT, Montreal, Canada, 2004

24. S. Carmignato, Accuracy of industrial computed tomography measurements: experimental results from an international comparison, CIRP Ann. Manuf. Technol. 61, 491$494(2012)$ 